\title{
Clinical decision making: what is the next step?
}

\author{
Ragesh Panikkath MD, DM, Deepa Panikkath MD
}

\section{CASE}

A 53-year-old man with history of bronchial asthma presented to the emergency department with fever and productive cough of two days duration. His respiratory rate was 18 /minute. His oxygen saturation was normal on room air. On physical examination, breath sounds were equal bilaterally. There were crepitations in the left infraaxillary and left infra-scapular area. There was also increased vocal fremitus with egophony in these areas. No bronchial breathing was heard. Laboratory results were significant for leukocytosis and increased serum creatinine compared to baseline. A PA chest radiograph is shown below.

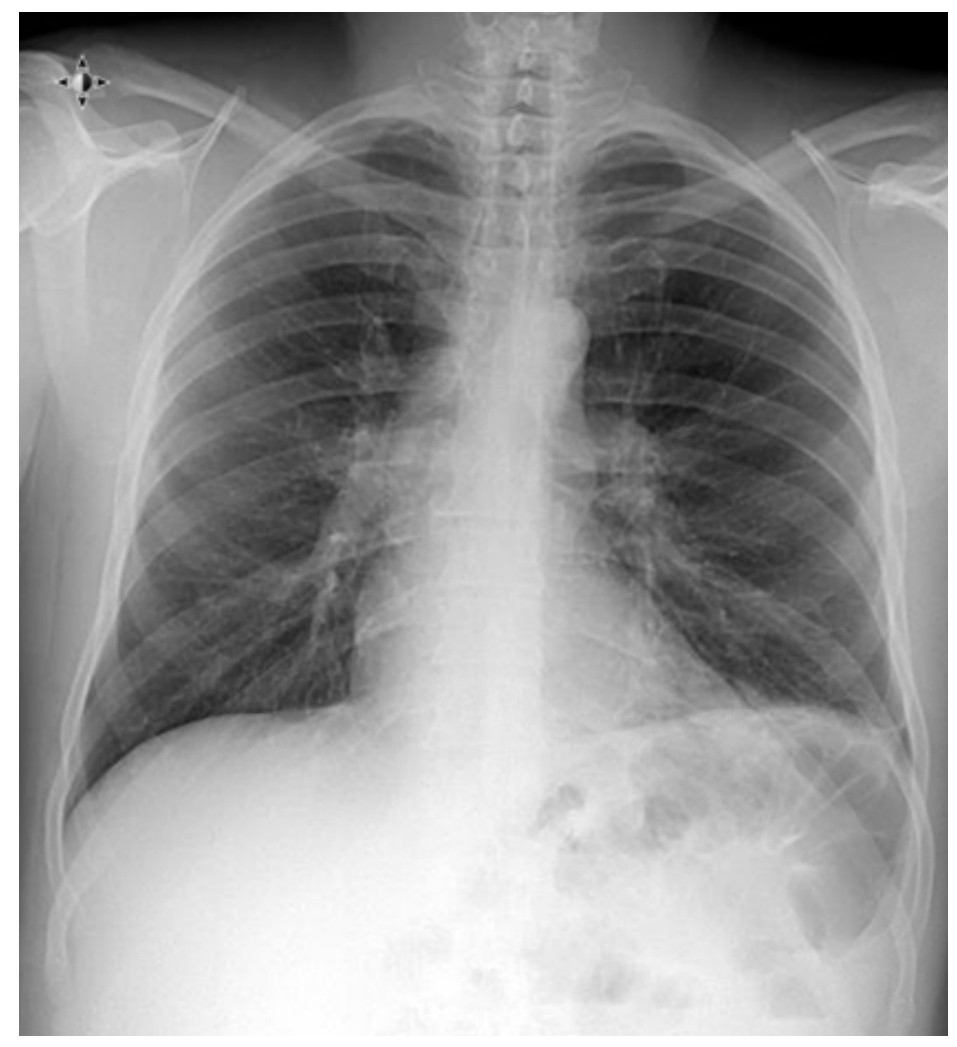

What is the next best step in management?
A. Order a CT scan of the chest
B. Order a troponin level
C. Order a d-dimer level
D. Order a lateral view chest $\mathrm{x}$-ray
E. Order a chest $\mathrm{x}$-ray taken after forced expiration

Corresponding author: Ragesh Panikkath MD, DM Contact Information: Ragesh.panikkath@ttuhsc.edu DOI: :10.12746/swrccc2014.0207.093 


\section{ANSWER}

The lateral view of the chest-x-ray is included below. It shows consolidation of the posterior segment of the left lower lobe. This was not evident in the PA film because of the retrocardiac and retrocolonic location. He was admitted to the hospital and was treated as community-acquired pneumonia. He improved and was discharged 2 days later.

Portable AP chest radiographs, although easy to obtain, may not disclose infiltrates in the retro-cardiac region, and, as seen in this case, PA films must also be scrutinized carefully in the setting of a significantly abnormal physical examination. This case illustrates the importance of the clinical examination and the lateral view chest $\mathrm{x}$-ray. Inexperienced physicians may find the lateral view intimidating and resort to more expensive imaging like computed tomography when a lateral chest x-ray provides the necessary information.

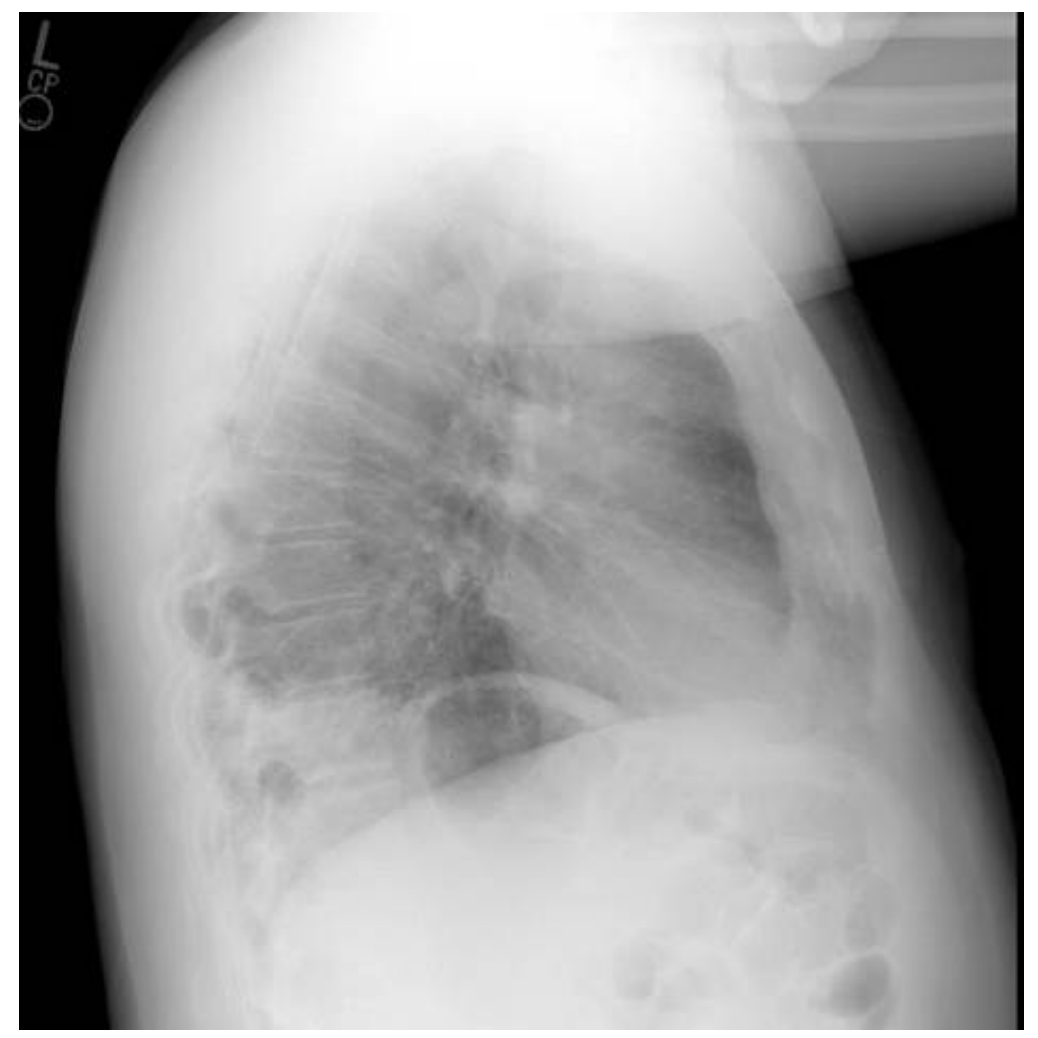

\section{REFERENCES}

1. Gaber KA, McGavin CR, Wells IP. Lateral chest X-ray for physicians. $J$ R Soc Med. 2005;98:310-2.
Author Affiliation: Ragesh Panikkath and Deepa Panikkath are residents in Internal Medicine at TTUHSC in Lubbock, TX.

Received: 05/07/2014

Accepted: 05/10/2014

Reviewers: Zachary Mulkey MD

Published electronically: 07/15/2014

Conflict of Interest Disclosures: none 